\title{
Development of Fluorescent Gelators and Detection of Explosives using Fibrous Xerogel Films
}

\author{
Kenji Hanabusa, ${ }^{* 1,2}$ Kazuki Harano,, Masafumi Fujisaki, ${ }^{3}$ Yasushi Nomura, ${ }^{3}$ \\ Masahiro Suzuki ${ }^{1}$ \\ 1 Interdisciplinary Graduate School of Science and Technology, Shinshu \\ University, Ueda, 386-8567 Japan \\ E-mail: hanaken@shinshu-u.ac.jp \\ ${ }^{2}$ Institute for Fiber Engineering, ICCER, Shinshu University, Ueda, 386-8567 \\ Japan \\ ${ }^{3}$ Faculty of Textile Science \& Technology, Shinshu University, Ueda, 386- \\ 8567 Japan
}

Summary: Fluorescent gelators containing stilbene segments were synthesized, and their gelation abilities were studied. Fibrous xerogel films were prepared from the gels on quartz plates, and their abilities to detect explosives were evaluated by the fluorescence quenching of the xerogel films upon exposure to saturated TNT or RDX vapor. The morphologies of the xerogel films were studied by TEM and DFM. The xerogel films that showed effective fluorescence quenching were found to have 3D fibrous networks with widths of several tens of nanometers and entangled fibrous aggregates with very uneven surfaces. The mechanism for detecting TNT was discussed from the viewpoint of HOMO and LUMO levels.

Keywords: gels; gelation; sensors; morphology; TEM

\section{Introduction}

Low-molecular-weight compounds that can physically gel water or organic solvents are called gelators. Gelators are gaining not only academic interest but also interest due to their practical applications. To the best of our knowledge, the first report regarding gelators was in 1942 when Yamamoto described the gelator 1,3:2,4-dibenzylidene-Dsorbitol $^{[1]}$. Papers on gelators were rare for the next half-century; however, research interest increased again during the first half of the 1990s. Combined with advances in the area of supramolecular chemistry, large numbers of reports on gelators have been published in recent years ${ }^{[2-20]}$. Gelators of low-molecular-weight compounds have unique characteristics including good solubility upon heating and the ability to induce the smooth gelation of liquids at low concentration. Gelators are also characterized by thermally reversible sol-gel transitions. These features can be attributed to 3D network structures, which are built up through noncovalent interactions such as hydrogen bonding, electrostatic interaction, van der Waals interaction, and $\pi-\pi$ interaction. Although several 
gelators have been already used as hardeners for spilled fluids and cooking oils, cosmetic materials, and thickeners for paint, the recent gelator research has focused on applications such as drug delivery in biomaterials ${ }^{[21,22]}$, scaffold materials for cells in tissue engineering $^{[23,24]}$, sensors ${ }^{[25-28]}$, templates for the synthesis of inorganic nanostructures ${ }^{[29-}$ ${ }^{33]}$, auxiliary agents for producing organic electronics ${ }^{[34,35]}$, and electrolytes that prevent liquid leakage ${ }^{[36]}$.

The recent rise of terrorism has become a matter of grave concern due the immense damage that can be caused by explosives. Thus, scientists and chemists in particular have developed methods for the rapid detection of explosives. Several methods have been practically used to detect explosives, including ion-mobility spectrometry, neutron backscattering, and explosive-detecting dogs. However, these methods still suffer from many problems such as the need for nonportable heavy instruments and technicians for operation (e.g., radiographers) and high cost. A simple approach that utilizes reagents to detect vapors in explosives is an attractive alternative that is small, lightweight, and low cost. Recently, Takeuchi et al. ${ }^{[3]}$ reported the detection of TNT vapor using the quenching of donor-acceptor-substituted stilbenes containing chiral binaphthyl groups. Liu et al. ${ }^{\text {[38] }}$ reported fluorescent sensor for TNT using electrospun nanofibrous films of porphyrin. Zang et al., ${ }^{[39]}$ Möllen et al., ${ }^{[40]}$, and Han et al. ${ }^{[41]}$ successfully detected TNT using carbazole backbone-based fluorescent compounds with amorphous morphologies due to oligomers, polymers, and dendrimers, respectively. Ajayaghosh et al. ${ }^{[42]}$ examined oligo(pphenylenevinylene)-based gelators for TNT detection. Bhattacharya et al. ${ }^{[43]}$ reported the detection of 2,4,6-trinitrophenol in solutions by monitoring the fluorescence quenching of a p-phenylenevinylene-based probe.

Over the last two decades, we have developed many low-molecular-weight gelators and proposed the concept of a "gelation-driving segment." This gelation-driving segment is expected to be a very useful tool for developing various types of gelators. ${ }^{[44]}$ In particular, functional groups such as hydroxyl and carboxyl groups, terminal double bonds, and halides are of special interest for the synthesis of functional gelators.

In this paper, we report the synthesis of fluorescent gelators based on the gelationdriving segment concept and employ fibrous xerogel films based on the gelators as chemosensors for the detection of explosives. One of the most important features of gelators is their formation of gels with self-assembled fibers instead of crystals. Xerogel films derived from gels have large surface areas due to the formation of fibrous aggregates, which is an advantage for sensor applications. In this study, we used stilbenes substituted 
with donor-acceptors in the 4,4'-positions, as reported by Takeuchi et al. ${ }^{[37]}$ We then synthesized fluorescent gelators by reacting the gelation-driving segments with these stilbenes. As oxidative explosives, we focused on TNT and RDX, the main components of plastic explosives.

\section{Experimental Section}

Instrumentation: Elemental analysis was performed with a Perkin-Elmer 240B analyzer. Infrared spectra were recorded on a Jasco FTIR-7300 spectrometer using $\mathrm{KBr}$ plate. Transmission electron microscopy (TEM) and dynamic force mode (DFM) of scanning probe microscope were done with a JEOL JEM-SS and a SII SPA-4001, respectively. UVvis and fluorescence spectra were recorded on a Jasco V-570UV/VIS/NIR and a Jasco FP6300. ${ }^{1} \mathrm{H}$ NMR spectra were taken in $\mathrm{CDCl}_{3}$ on a Bruker AVANCE 400 spectrometer and the chemical shifts were recorded in ppm $(\delta)$ downfield from TMS. Thickness of films was measured by a Yamato Scientific Alpha-Step D-600.

\section{Synthesis of Fluorescent Gelators:}

Solvents, triphenylphosphine, and tetrabutylammonium iodide were purchased from Wako Pure Chemical Industries. Wakogel ${ }^{\circledR}$ C-200 (Wako Pure Chemical Industries) was used as silica gel for column chromatography. 4-Cyanobenzyl bromide, 4-nitrobenzaldehyde, 4nitrobenzylbromide, and 3,5-bis(trifluoromethyl)benzaldehyde were purchased from Tokyo Kasei. L-Isoleucine and $N^{\varepsilon}$-lauroyl-L-lysine were granted from Ajimonoto Co. Ltd. trans-4-(4-Nitrostyryl)benzonitrile: This compound was prepared by the method described in the literature ${ }^{[45]}$. A mixture of $9.8 \mathrm{~g}(0.05 \mathrm{~mol})$ of 4-cyanobenzyl bromide and $13.1 \mathrm{~g}(0.05 \mathrm{~mol})$ of triphenylphosphine in $250 \mathrm{~mL}$ of toluene was refluxed for $6 \mathrm{~h}$. After evaporating toluene, the residue was dried in vacuo. 4-Nitrobenzaldehyde (7.55 g, 0.05 mol), $18.45 \mathrm{~g}$ (0.05 mol) of tetrabutylammonium iodide, and $400 \mathrm{~mL}$ of $\mathrm{CH}_{2} \mathrm{Cl}_{2}$ were added to the residue. The resulting solution was added dropwise to a saturated solution of potassium carbonate in $300 \mathrm{~mL}$ of water, and then refluxed overnight. The separated organic layer after standing was washed with $100 \mathrm{~mL}$ of water twice, and dried with $\mathrm{MgSO}_{4}$, followed by evaporation. A small portion of trans-isomer was obtained by recrystallization from chloroform. The trans-isomer was obtained in a yield of $0.5 \mathrm{~g}$. The cis-isomer in the filtrate was purified by column chromatography on silica gel (elution 
with chloroform; $\mathrm{Rf}=0.72$ ). The diameter inside and length of the column were 40 and $540 \mathrm{~mm}$, respectively. The cis-isomer was obtained in a yield of $8.3 \mathrm{~g}(66 \%)$. The cisisomer was dissolved in a minimum quantity of chloroform including $46 \mathrm{mg}$ of iodine, followed by irradiated with high-pressure mercury lamp for $30 \mathrm{~min}$. The trans-isomer was precipitated instantly as yellow needle crystals by irradiation. trans-4-(4Nitrostyryl)benzonitrile was isolated in a yield of $8.4 \mathrm{~g}(67 \%)$.

$\operatorname{IR}\left(\mathrm{KBr}, \mathrm{cm}^{-1}\right): 3036(\mathrm{C}=\mathrm{C}), 2222(\mathrm{~N} \equiv \mathrm{C}), 1602$ (aromatic); ${ }^{1} \mathrm{H} \mathrm{NMR}\left(\mathrm{CDCl}_{3}, 400 \mathrm{MHz}\right)$ : $\delta 8.26(\mathrm{~d}, \mathrm{~J}=8.84,2 \mathrm{H}), 7.67(\mathrm{~d}, \mathrm{~J}=6.84,2 \mathrm{H}) 7.66(\mathrm{~d}, \mathrm{~J}=7.16,2 \mathrm{H}), 7.62(\mathrm{~d}, \mathrm{~J}=8.702 \mathrm{H})$, 7.31 (d, J = 5.52, 1H), 7.21 (d, J = 3.76, 1H); Found: C 71.41, H 4.58, N 11.81\%. Calcd for $\mathrm{C}_{15} \mathrm{H}_{10} \mathrm{~N}_{2} \mathrm{O}_{2}$ : C 71.99, $\mathrm{H}$ 4.03, N 11.19\%.

trans-4-(4-Aminostyryl)benzonitrile: A mixture of $1.47 \mathrm{~g}(6.0 \mathrm{mmol})$ of trans-4-(4nitrostyryl)benzonitrile and $5.69 \mathrm{~g}(0.03 \mathrm{~mol})$ of $\mathrm{SnCl}_{2}$ in $40 \mathrm{~mL}$ of ethanol was stirred at $70^{\circ} \mathrm{C}$ for $18 \mathrm{~h}$ under an argon atmosphere. The cooled mixture was poured to $\sim 30 \mathrm{~g}$ of ice and then $5.0 \mathrm{~g}$ of $\mathrm{NaHCO}_{3}$ was added by portions. The matter was extracted with ethyl acetate and recrystallized from ethanol. The obtained crude product was purified by column chromatography on silica gel (elution with chloroform; $\mathrm{Rf}=0.45$ ). Recrystallization from ethanol gave trans-4-(4-aminostyryl)benzonitrile in a yield of 0.85 g (64\%).

$\operatorname{IR}\left(\mathrm{KBr}, \mathrm{cm}^{-1}\right)$ : $3436\left(\mathrm{NH}_{2}\right), 3020(\mathrm{C}=\mathrm{C}), 2923(\mathrm{C} \equiv \mathrm{N}), 1619$ (Aromatic); ${ }^{1} \mathrm{H}$ NMR $\left(\mathrm{CDCl}_{3}\right.$, $400 \mathrm{MHz}): \delta 7.60(\mathrm{~d}, \mathrm{~J}=8.6,2 \mathrm{H}), 7.53(\mathrm{~d}, \mathrm{~J}=8.4,2 \mathrm{H}), 7.36(\mathrm{~d}, \mathrm{~J}=8.6,2 \mathrm{H}), 7.14(\mathrm{~d}, \mathrm{~J}=$ 16.3, 1H), 6.90 (d, J = 16.3, 1H), 6.69 (d, J = 8.5, 2H) ; Found: C 81.61, H 5.58, N 12.81\%. Calcd for $\mathrm{C}_{15} \mathrm{H}_{12} \mathrm{~N}_{2}$ : C 81.79, H 5.49, N 12.72\%.

trans-4-(4-Nitrostyryl)-3,5-bis(trifluoromethyl)benzene: This compound was prepared from 4-nitrobenzylbromide and 3,5-bis(trifluoromethyl)benzaldehyde by the similar procedure described above. Yield: $58 \%, \mathrm{Rf}=0.8$ (silica gel, chloroform).

IR(KBr, $\mathrm{cm}^{-1}$ ): 3036 (C=C), 1602 (aromatic); Found: C 52.98, H 2.22, N 3.84\%. Calcd for $\mathrm{C}_{16} \mathrm{H}_{9} \mathrm{~F}_{6} \mathrm{NO}_{2}$ : C 53.20, H 2.51, N 3.88\%.

trans-4-(4-Aminostyryl)-3,5-bis(trifluoromethyl)benzene: This compound was obtained by reduction of trans-4-(4-nitrostyryl)-3,5-bis(trifluoromethyl)benzene by the similar method described above. Yield: 43\%, Rf $=0.5$ (silica gel, chloroform).

IR(KBr, $\left.\mathrm{cm}^{-1}\right)$ : 3436, $\left(\mathrm{NH}_{2}\right), 3020(\mathrm{C}=\mathrm{C}), 1619$, (aromatic); Found: C 52.98, H 2.22, N 
3.84\%. Calcd for $\mathrm{C}_{16} \mathrm{H}_{11} \mathrm{~F}_{6} \mathrm{~N}$ : C 53.20, H 2.51, N 3.88\%.

Fluorescence gelator 1: $N$-Carbobenzyloxy-L-isoleucylaminooctadecane ${ }^{[36]}$ (51.68 g, $0.10 \mathrm{~mol}$ ) was hydrogenated in the presence of Pd-C in $500 \mathrm{~mL}$ of 1-propanol for $5 \mathrm{~h}$ at room temperature under a hydrogen atmosphere. After confirming the complete removal of protecting group by TLC (chloroform : methanol : acetic acid = 95:5:1 as volume ratio), the solution was filtered off. The filtrate was evaporated and recrystallization from $300 \mathrm{~mL}$ of ligroin provided $35.3 \mathrm{~g}$ (92\%) of L-isoleucylaminooctadecane. A solution of $7.0 \mathrm{~g}$ (18 mmol) of L-isoleucylaminooctadecane and $4.9 \mathrm{~mL}$ (36 mmol) of triethylamine in $400 \mathrm{~mL}$ of dry THF was cooled in ice-water bath, and then $5.44 \mathrm{~g}$ (19.2 mmol) of 11bromoundecanoyl chloride was added dropwise. The matter was stirred for $1 \mathrm{~h}$ in icewater bath, followed by for $4 \mathrm{~h}$ at room temperature. A filtrate without $\mathrm{NEt}_{3} / \mathrm{HCl}$ salt was evaporated and recrystallized from ethanol/ether. N-11-Bromoundecanoyl-Lisoleucylaminooctadecane was obtained in a yield of $9.25 \mathrm{~g}$ (82\%). A mixture of $1.01 \mathrm{~g}$ (1.6 mmol) of $N$-11-bromoundecanoyl-L-isoleucylaminooctadecane, $0.48 \mathrm{~g}$ (3.5 mmol) of potassium carbonate, and $0.50 \mathrm{~g}(3.0 \mathrm{mmol})$ of potassium iodide in $10 \mathrm{~mL}$ of dry DMF was heated to obtain a homogeneous solution. To the solution was added $0.18 \mathrm{~g}$ ( 0.8 mmol) of trans-4-(4-aminostyryl)benzonitrile, followed by stirred at $40^{\circ} \mathrm{C}$ overnight under an argon atmosphere. The mixture was poured to iced water and the precipitated matter was filtered with suction, washed with water, and dried. Recrystallization from ethanol gave a crude product, which was purified by column chromatography on silica gel (elution with chloroform; $\mathrm{Rf}=0.10$ ). The diameter inside and length of the column were 25 and $400 \mathrm{~mm}$, respectively. The compound 1 was obtained by recrystallization from methanol in a yield of $0.65 \mathrm{~g}(62 \%)$.

IR(KBr, cm$\left.{ }^{-1}\right)$ : 3288 (amide), $2918\left(\mathrm{CH}_{2}\right)$, 1635 (C=O); Found: C 76.52, H 11.45, N 11.34\%. Calcd for $\mathrm{C}_{85} \mathrm{H}_{148} \mathrm{~N}_{6} \mathrm{O}_{4}$ : C 77.45, H 11.32, N 6.38\%.

Fluorescence gelator 2: A mixture of 0.74 g $(3.4$ mmol $)$ of trans-4-(4aminostyryl)benzonitrile and $2.0 \mathrm{~g} \quad(3.4 \mathrm{mmol})$ of $N$-(6-isocyanato-trimethylhexylaminocarbonyl)-L-isoleucylaminooctadecane ${ }^{[46]}$ in $30 \mathrm{~mL}$ of dry toluene was stirred at $85^{\circ} \mathrm{C}$ overnight under an argon atmosphere. After evaporating toluene in vacuo, the residue was recrystallized from ethanol. The obtained crude product was purified by column chromatography on silica gel (elution with chloroform; $\mathrm{Rf}=0.20$ ). The compound 2 was obtained by recrystallization from ethanol in a yield of $2.2 \mathrm{~g}(80 \%)$. 
IR(KBr, $\left.\mathrm{cm}^{-1}\right)$ : $3338(\mathrm{~N}-\mathrm{H}$, urea), $2921(\mathrm{C} \equiv \mathrm{N}), 1629$ (C=O, amide); Found: C 73.42, H 9.78, N 10.66\%. Calcd for $\mathrm{C}_{60} \mathrm{H}_{80} \mathrm{~N}_{6} \mathrm{O}_{3}$ : C 73.85, H 9.92, N 10.33\%.

Fluorescence gelator 3: This compound was prepared from $0.74 \mathrm{~g}$ (3.4 mmol) of trans-4(4-aminostyryl)benzonitrile and $1.78 \mathrm{~g}(3.4 \mathrm{mmol})$ of $N^{\alpha}$-(6-isocyanato-trimethylhexylaminocarbonyl)- $N^{\varepsilon}$-lauroyl-L-lysine ethyl ester ${ }^{[47]}$ by the similar procedure described in fluorescence gelator 2. Yield: $2.00 \mathrm{~g}$ (82\%), $\mathrm{Rf}=0.2$ (silica gel, chloroform).

IR(KBr, cm $\left.{ }^{-1}\right)$ : $3338(\mathrm{~N}-\mathrm{H}$, urea), $2921(\mathrm{C} \equiv \mathrm{N}), 1733$ (C=O, ester), 1629 (C=O, amide); Found: C 68.84, H 8.97, N 11.34\%. Calcd for $\mathrm{C}_{43} \mathrm{H}_{64} \mathrm{~N}_{6} \mathrm{O}$ : C 69.32, H 8.66, N 11.28\%.

Fluorescence gelator 4: This compound was prepared from $1.13 \mathrm{~g}$ (3.4 mmol) of trans-4(4-aminostyryl)-3,5-bis(trifluoromethyl)benzene and $2.0 \mathrm{~g}(3.4 \mathrm{mmol})$ of $N^{\alpha}$-(6isocyanato-trimethyl-hexylaminocarbonyl)- $N^{\varepsilon}$-lauroyl-L-lysine ethyl ester by the similar procedure described in fluorescence gelator 2. Yield: $1.56 \mathrm{~g}$ (50\%), Rf $=0.1$ (silica gel, chloroform).

IR(KBr, cm $\left.{ }^{-1}\right)$ : $3339(\mathrm{~N}-\mathrm{H}$, urea), $2929(\mathrm{C} \equiv \mathrm{N}), 1626$ (C=O, amide); Found: C 79.76, H 6.57, N 6.14\%. Calcd for $\mathrm{C}_{51} \mathrm{H}_{79} \mathrm{~F}_{6} \mathrm{~N}_{5} \mathrm{O}_{3}$ : C 79.97, H 6.71, N 6.22\%.

TNT and RDX: These explosives were prepared precisely according to the literature ${ }^{[48-50]}$.

Gelation test: Gelation test was carried out by upside-down test tube method. A typical procedure is as follows: A weighed sample and $1 \mathrm{~mL}$ of solvent in a septum-capped test tube with internal diameter of $14 \mathrm{~mm}$ was heated until the solid dissolved. The resulting solution was cooled at $25^{\circ} \mathrm{C}$ for $1 \mathrm{~h}$ and then the gelation was checked visually. When no fluid ran down the wall of the test tube upon inversion of the test tube, we judged it to be gel. The gelation ability was evaluated by the minimum gel concentration, which is the minimum concentration of a gelator necessary for gelation at $25^{\circ} \mathrm{C}$. The unit is $\mathrm{g} \mathrm{L}^{-1}$ (gelator/solvent).

Preparation of Xerogel Films as Sensors: Xerogel films as chemosensor were prepared by directly drop-casting method. A typical procedure is as follows: The warm ethanol solution of compound $1\left(5.0 \times 10^{-4} \mathrm{M}\right)$ was drop-casted on a quartz plate of $15 \mathrm{~mm}(\mathrm{~W}) \times 2$ $\mathrm{mm}(\mathrm{D})$ x $50 \mathrm{~mm}(\mathrm{H})$, followed by spin-coated, and then dried in vacuo. Thickness of film 
was controlled by the number of drops. The spin-coat was controlled to be $1500 \mathrm{rpm}$.

Theoretical Calculations: The trans-4-(4-aminostyryl)benzonitrile, trans-4-(4aminostyryl)- 3,5-bis(trifluoromethyl)benzene, and TNT were geometry-optimized in gas phase using the B3LYP/6-31G* method, and the HOMO and LUMO levels of the molecules were calculated.

\section{Results and Discussion}

\section{Fluorescent Compounds and Gelation Abilities}

Compounds 1-4 containing trans-4-(4-aminostyryl)benzonitrile or trans-4-(4aminostyryl)-3,5-bis(trifluoromethyl)benzene were prepared as candidate fluorescent gelators (Scheme 1). Compounds 1 and 2 were obtained by the alkylation reaction of $\mathrm{N}$ (11-bromoundecyldecanoyl)-L-isoleucylaminooctadecane with 1 and by the addition of $\mathrm{N}$ (6-isocyanato-trimethyl-hexylaminocarbonyl)-L-isoleucylaminooctadecane, respectively. Compound 3 was obtained by the addition of $N^{\alpha}$-(6-isocyanato-trimethylhexylaminocarbonyl)- $N^{\varepsilon}$-lauroyl-L-lysine ethyl ester. Compound 4 was prepared from $N$ (6-isocyanato-trimethyl-hexylaminocarbonyl)-L-isoleucylaminooctadecane and trans-4(4-aminostyryl)-3,5-bis(trifluoromethyl)benzene. $\quad \mathrm{N}$-(11-Bromoundecyldecanoyl)-Lisoleucylaminooctadecane, $\quad N$-(6-Isocyanato-trimethyl-hexylaminocarbonyl)-L-isoleucylaminooctadecane, and $N^{\alpha}$-(6-isocyanato-trimethyl-hexylaminocarbonyl)- $N^{\varepsilon}$-lauroyl-Llysine ethyl ester, which we developed, were used as the gelation-driving segments. 

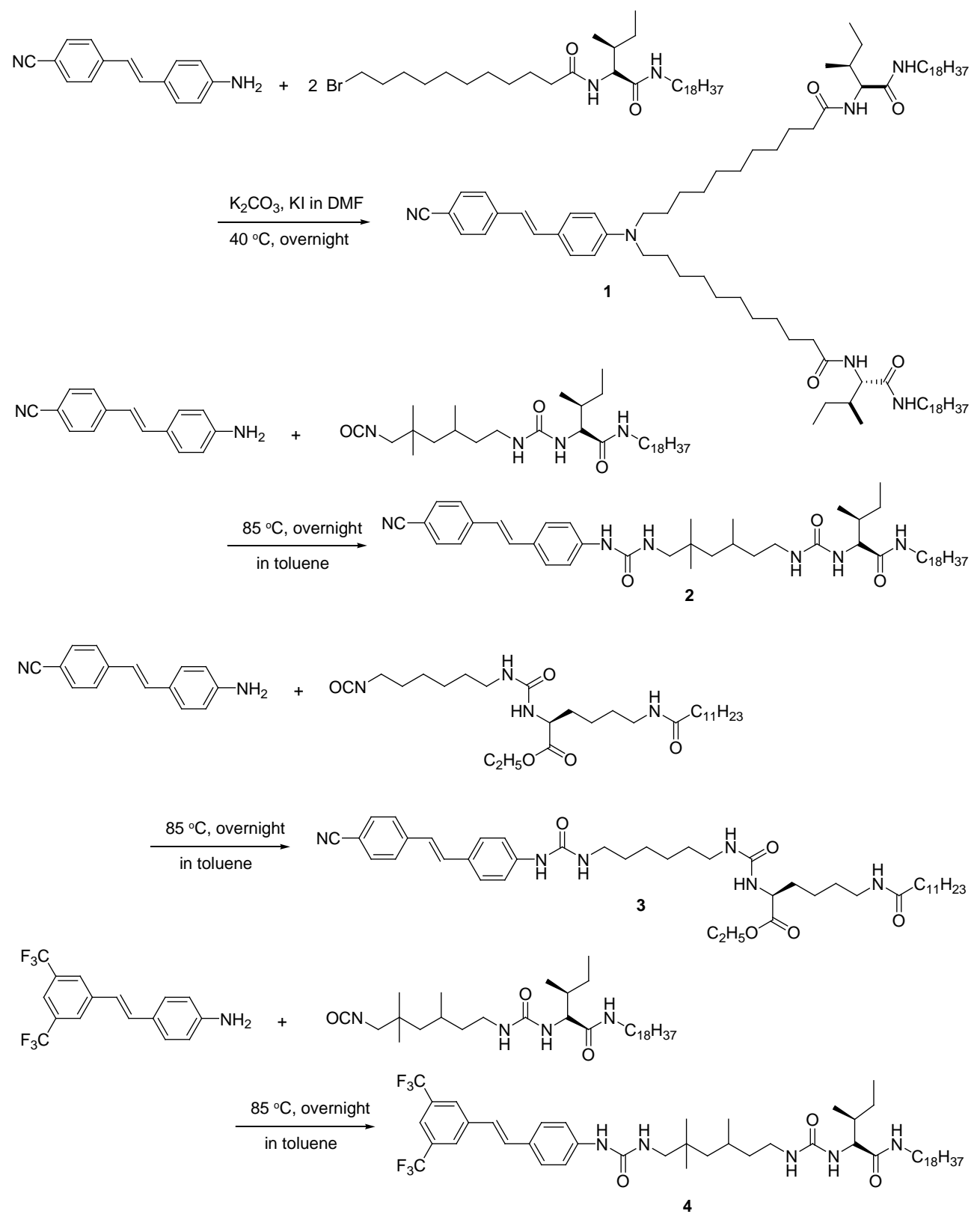

Scheme 1. Synthesis of fluorescent compounds 1-4.

Gelation tests were carried out using the upside-down test-tube method. The results of the gelation tests using ethanol, toluene, hexane, and chloroform (Table 1) indicated that (i) compounds 1, 2, and $\mathbf{4}$ are gelators that can gel ethanol and toluene, (ii) compound $\mathbf{3}$ has no gelation ability, and (iii) all compounds are miscible in chloroform. The lack of gelation ability in $\mathbf{3}$ may arise from the absence of an appropriate hydrophile-lipophile balance; the ethyl ester part in $\mathbf{3}$ may increase its solvophilic character. The gelationdriving segments including L-isoleucine residue are thought to be very useful in the syntheses of functional gelators. 
Table 1. Gelation tests of fluorescent compounds 1-4 ${ }^{\text {a }}$.

\begin{tabular}{lcclc}
\hline Solvent & $\mathbf{1}$ & $\mathbf{2}$ & $\mathbf{3}$ & $\mathbf{4}$ \\
\hline Ethanol & $\mathrm{GO}(40)$ & $\mathrm{GO}(40)$ & $\mathrm{S}$ & $\mathrm{GO}(10)$ \\
Toluene & $\mathrm{GTL}(10)$ & $\mathrm{GTL}(20)$ & $\mathrm{S}$ & $\mathrm{GT}(20)$ \\
Hexane & $\mathrm{I}$ & $\mathrm{GO}(20)$ & $\mathrm{I}$ & $\mathrm{S}$ \\
Chloroform & $\mathrm{S}$ & $\mathrm{S}$ & $\mathrm{S}$ & $\mathrm{S}$ \\
\hline
\end{tabular}

${ }^{a}$ GT: Transparent gel. GTL: Translucent gel. GO: Opaque gel. S: Soluble. I: Almost insoluble. The values mean the minimum gel concentrations at $25^{\circ} \mathrm{C}$; the units are $\mathrm{g} \mathrm{L}^{-1}$ (gelator/solvent).

\section{Fluorescent Chemosensors of Xerogel Films for Detecting TNT}

The UV-vis absorption spectra and fluorescence spectra of gelators 1, 2, and 4 in chloroform are shown in Figure 1. The absorption peaks of 1, 2, and $\mathbf{4}$ are at 398, 349, and $343 \mathrm{~nm}$, respectively. The peak at $349 \mathrm{~nm}$ for $\mathbf{2}$ is blue shifted by $49 \mathrm{~nm}$ compared with $\mathbf{1}$. This blue shift is explained by accounting for the strong electron-withdrawing inductive effect of the carbonyl group, which participates in a urea bond connecting trans-4-(4aminostyryl)benzonitrile and the gelation-driving segment. The small $6 \mathrm{~nm}$ blue shift of 4 compared with $\mathbf{2}$ is likely attributed to two electron-withdrawing trifluoromethyl groups in the trans-4-(4-aminostyryl)-3,5-bis(trifluoromethyl)benzene segment. The fluorescence peaks of 1, 2, and 4 are at 503, 467, and $425 \mathrm{~nm}$, according to the Stokes shift. TNT was detected by monitoring the fluorescence quenching of these fluorescence $\lambda_{\max }$ peaks.

Xerogel chemosensors were prepared by directly drop-casting the gels or solutions onto quartz plates, followed by spin-coating and drying in vacuo. Gelation by lowmolecular-weight compounds occurs via the self-aggregation of molecules driven by noncovalent bonding, resulting in the formation of fibrous aggregates. The fibrous aggregates ultimately form a 3D network structure in which solvent molecules are trapped. Thus, nanosized fibrous aggregates inevitably form during the initial stage of gelation. Hence, it is reasonable to assume that the fibrous aggregates will be fixed in xerogel films prepared from low-molecular-weight gelators. The large surface area resulting from the formation of fibrous aggregates is an advantage for sensor applications. The sensing abilities of the xerogels were studied by exposing the xerogel films to TNT-saturated vapor. 

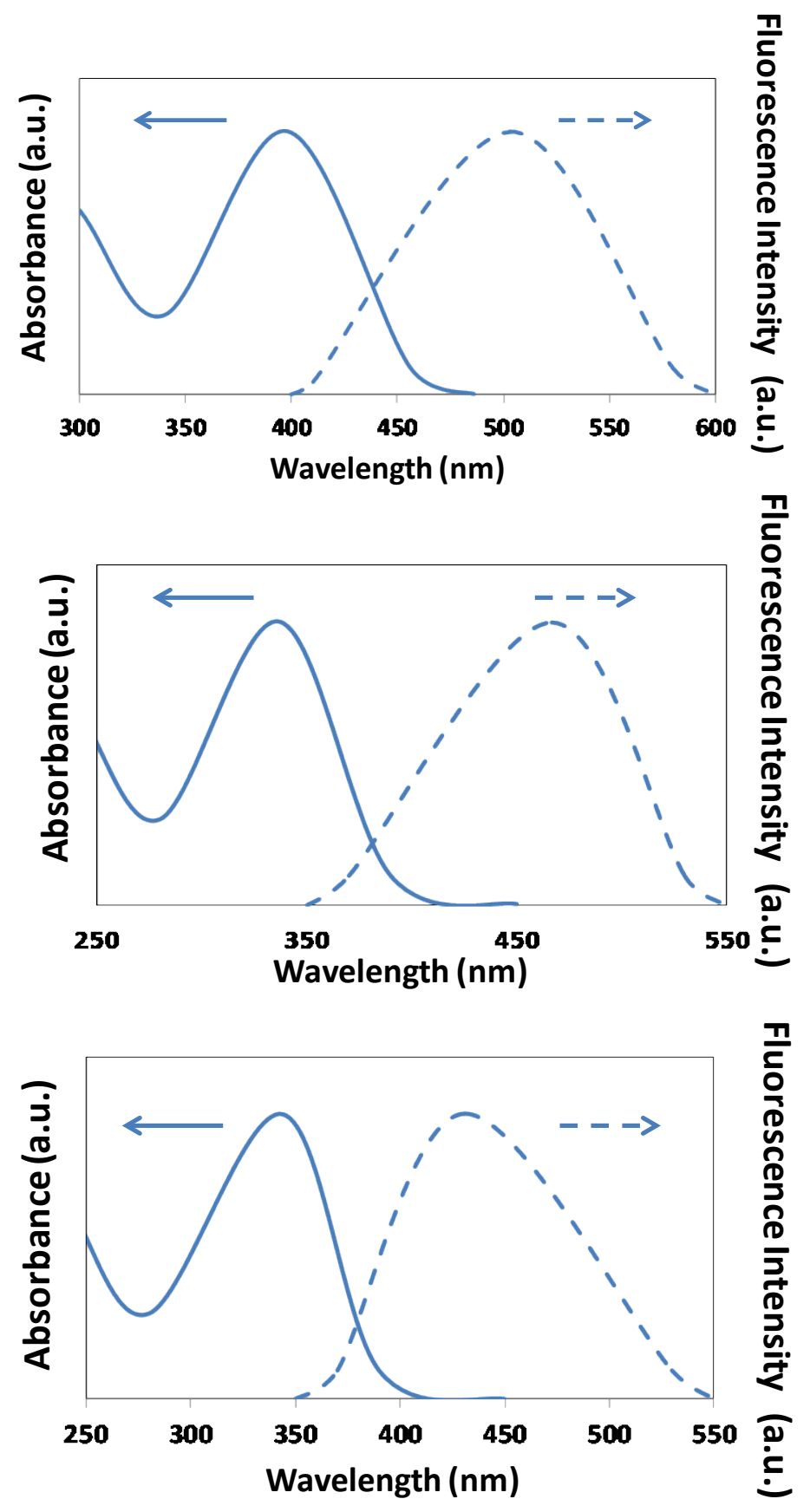

Figure 1. Absorption and fluorescence spectra of $\mathbf{1}, \mathbf{2}$, and $\mathbf{4}$ (from top to bottom).

Xerogel films were prepared by directly drop-casting the ethanol gels. Figure 2 shows the fluorescence quenching of the xerogel film (thickness, 32 nm) of gelator 1 upon exposure to saturated TNT vapor (vapor pressure, $\sim 5.0 \mathrm{ppb}$ ) for different time periods. About 72\% and 95\% quenching were observed after exposure to TNT for 2 and $20 \mathrm{~min}$, respectively. Nearly the same behavior was observed for the xerogel film (thickness, 32 $\mathrm{nm}$ ) of gelator 2 (Figure 3); the fluorescence quenching for 2 were $~ 69 \%$ and $72 \%$ for 2 and 20 min, respectively. Note that the microcrystalline film prepared by drop-casting the 
ethanol solution of trans-4-(4-aminostyryl)benzonitrile (thickness, 32 nm) showed 8\% and 26\% quenching after 2 and $20 \mathrm{~min}$, respectively. Moreover, the microcrystalline film prepared from the ethanol solution of compound 3, which could not gel ethanol (see Table 1), showed $\sim 11 \%$ and $27 \%$ quenching after 2 and $20 \mathrm{~min}$, respectively. We studied the quenching of gelator 1 against other aromatic compounds. When the xerogel films of gelator 1 were exposed on saturated vapors of $p$-dichlorobenzene, $p$-dicyanobenzene, and p-dimethoxybenzene, it showed $\sim 30 \%, 28 \%$, and 18\% quenching after $20 \mathrm{~min}$, respectively. Combining the results of gelators 1, 2, and 3 and trans-4-(4aminostyryl)benzonitrile, we can conclude that the xerogel films formed from lowmolecular-weight gelators are very effective chemosensors.

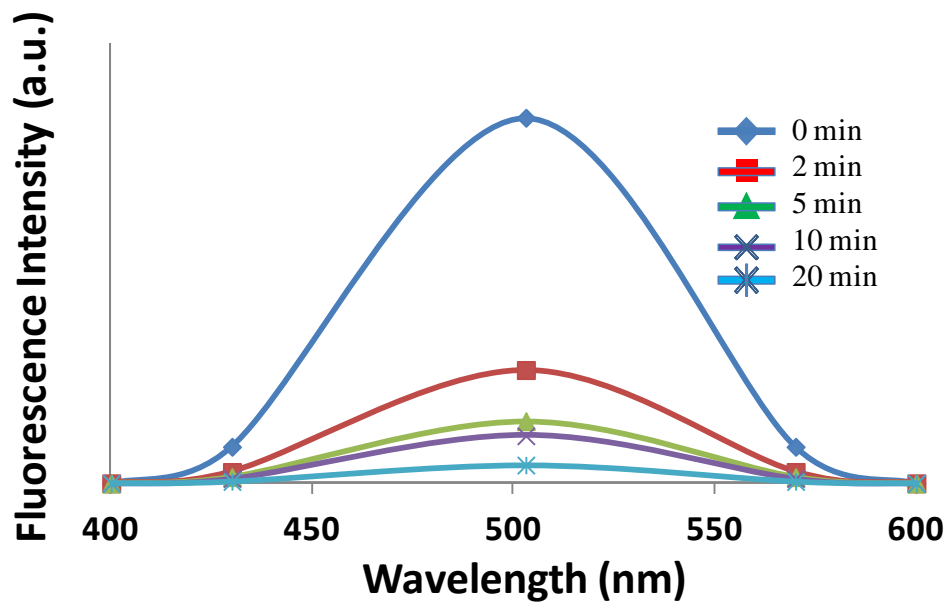

Figure 2. Fluorescence spectra of xerogel film of $\mathbf{1}$ casted from ethanol solution upon exposure to saturated TNT vapor for different time periods.

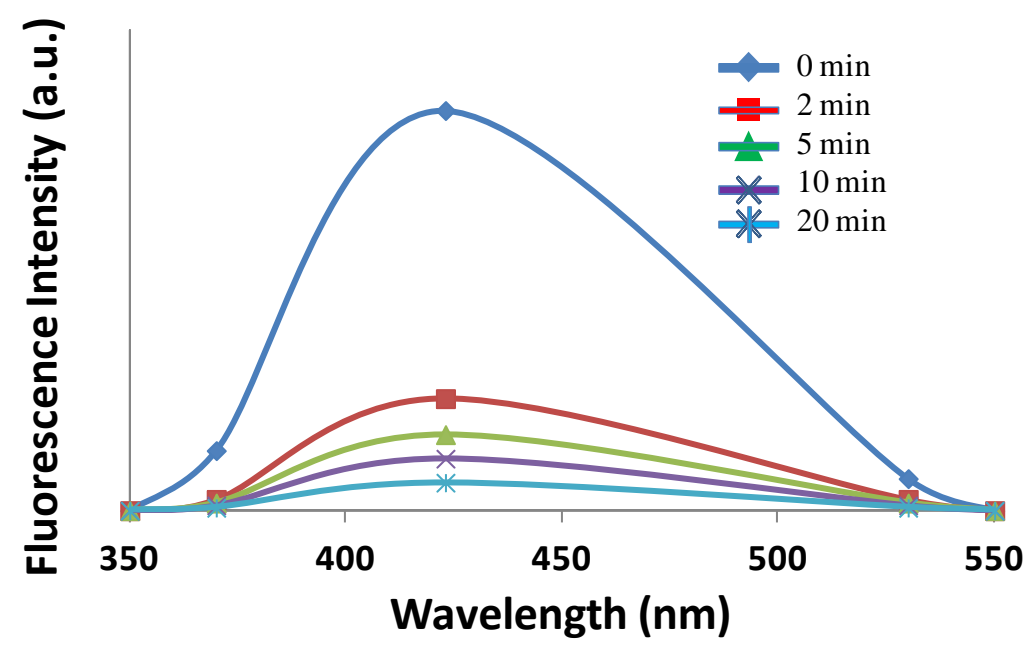


Figure 3. Fluorescence spectra of xerogel film of $\mathbf{2}$ casted from ethanol solution upon exposure to saturated TNT vapor for different time periods.

To ascertain whether the fibrous aggregates effectively acted as chemosensors, quenching was compared among three films prepared from gelator 2: two xerogel films formed from ethanol and toluene, and a microcrystalline film from chloroform solution. Figure 4 shows the fluorescence quenching of the xerogel films (thickness, $57 \mathrm{~nm}$ ) and the microcrystalline film (thickness, $\sim 57 \mathrm{~nm}$ ). Significant decreases in quenching efficiency upon exposure to TNT vapor were clearly observed in the xerogel films formed from ethanol and toluene gels compared with the film formed from chloroform solution. This indicates that the xerogel itself is more sensitive over the microcrystalline film, because of the wide surface area of xerogel.

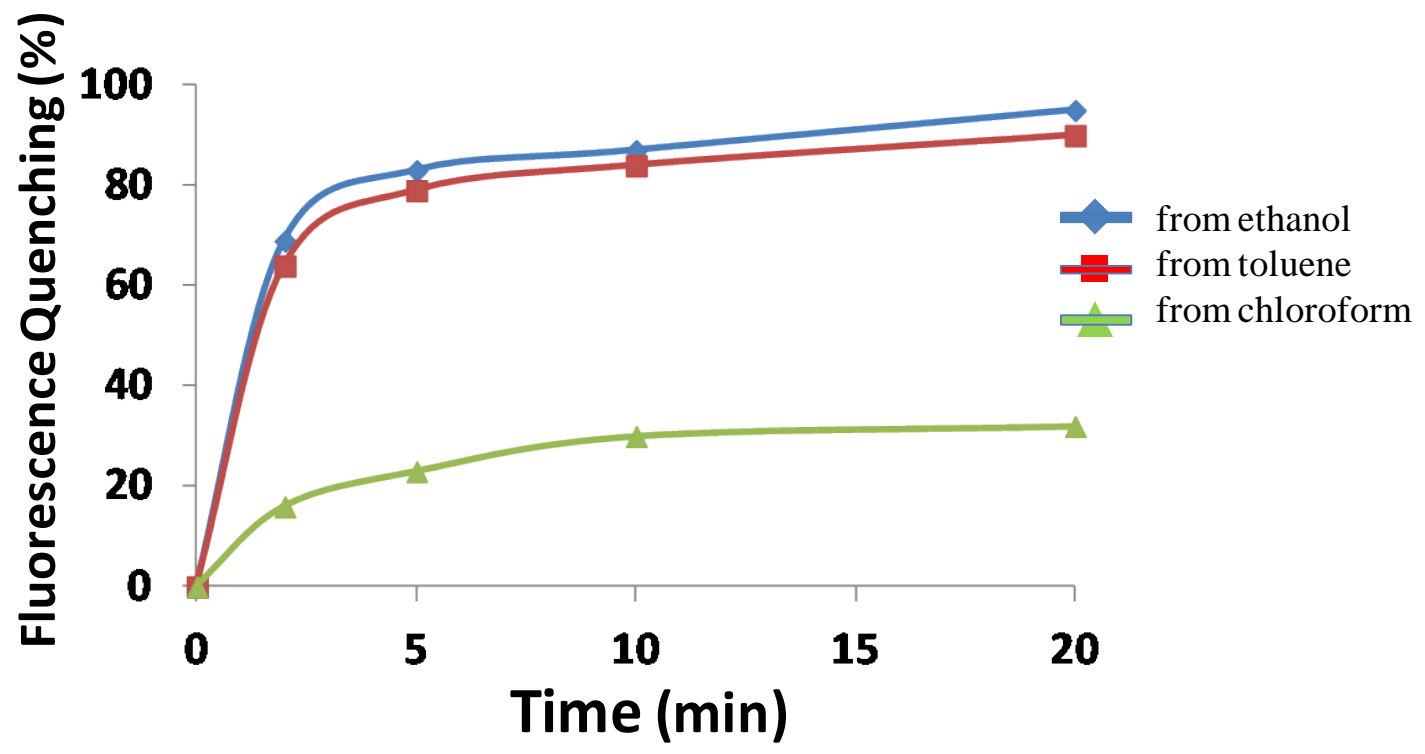

Figure 4. Fluorescence quenching of films of 2 casted from ethanol, toluene, and chloroform solutions, when exposed to saturated TNT vapor for different time periods.

The relationship between the thickness of the xerogel film and the quenching efficiency upon exposure to TNT was studied. Figure 5 shows the fluorescence quenching of xerogel films of gelator 1 with different thicknesses (32-2590 nm) after 2 min of exposure to TNT. The fluorescence quenching efficiency decreased distinctly with increasing film thickness. The quenching efficiency decreased from $72 \%$ in for a film thickness of $32 \mathrm{~nm}$ to $21 \%$ for a thickness of $2590 \mathrm{~nm}$. This result can be explained by considering the residual molecules deep in the thick films, which are not quenched by 
exposure to TNT. Thus, we can reasonably assume thinner films exhibit more effective fluorescence quenching. It should be mentioned that when the xerogel film thickness is less than $20 \mathrm{~nm}$, the fluorescence is too weak to detect.

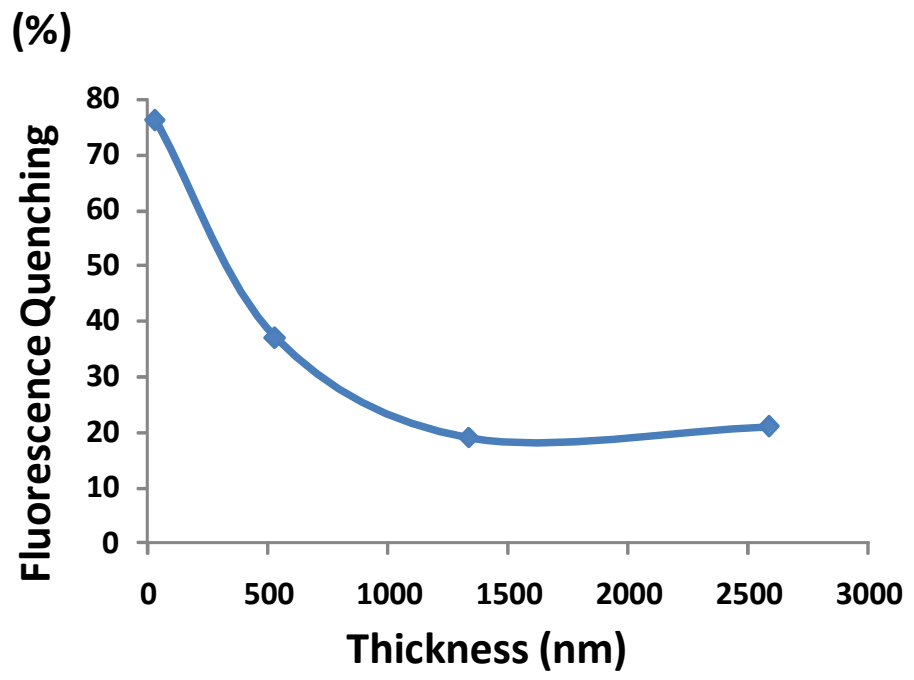

Figure 5. Fluorescence quenching and thickness of xerogel films of $\mathbf{1}$.

Quenching after the passage of $2 \mathrm{~min}$, the thickness from $32 \mathrm{~nm}$ to $2590 \mathrm{~nm}$.

\section{Fluorescent Chemosensors of Xerogel Films for Detecting RDX}

We studied the detection of nonaromatic explosive RDX (hexahydro-1,3,5-trinitro-1,3,5triazine). Figure 6 shows the fluorescence quenching of the xerogel films (thickness, 32 $\mathrm{nm}$ ) of gelators 1-4 upon exposure to saturated RDX vapor for different time periods. The xerogel films were prepared by drop-casting the toluene gels. Despite the very low vapor pressure $\left(\sim 5 \times 10^{-3} \mathrm{ppb}\right)$ of saturated RDX, moderately significant decreases in quenching efficiency were observed for the xerogel films of gelators 2 and 4; 60\% decreases in quenching efficiency were observed after $20 \mathrm{~min}$ for both films. This result is surprising because unlike TNT, RDX has no absorption in the visible light region. The contact of RDX vapor on the xerogel films and the subsequent oxidative decomposition of fluorescence gelators may be attributed to the fluorescence quenching. The cause for quenching by RDX requires further investigation considering the HOMO-LUMO energy level, excitation energy, and energy of ionization. 


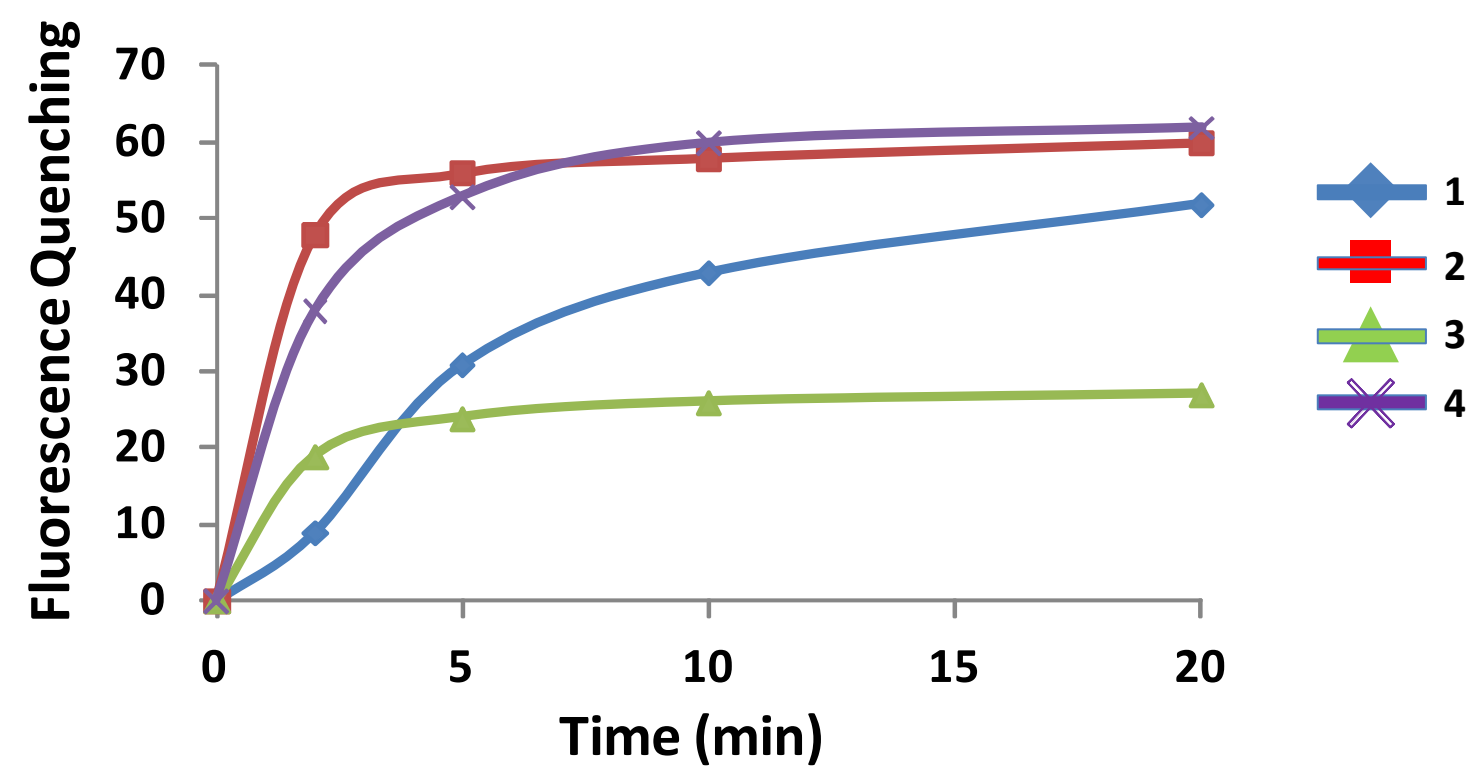

Figure 6. Fluorescence quenching of films of 1-4 casted from toluene gels, when exposed to saturated RDX vapor for different time periods.

\section{Morphologies of Fibrous Xerogel Films}

We used transmission electron microscopy (TEM) and dynamic force mode (DFM) of scanning probe microscopy to characterize the morphologies of the films. Figure 7 shows the TEM images of the films prepared from trans-4-(4-aminostyryl)benzonitrile, 1, 2, and 3. The xerogel films of fluorescent gelators 1 and $\mathbf{2}$, which exhibited effective quenching when exposed to TNT, exhibit 3D networks of juxtaposed and interlocked fibers with widths of several tens of nanometers. On the other hand, the films of trans-4-(4aminostyryl)benzonitrile and compound 3, which could not gel ethanol, exhibited crystalline nanostructures. The DFM images of the films also confirmed the fibrous and crystalline nanostructures (Figure 8). Dense entangled fibrous aggregates with very uneven surfaces were observed in the xerogel films of fluorescent gelators $\mathbf{1}$ and 2 . In contrast, the surfaces of the films of trans-4-(4-aminostyryl)benzonitrile and compound 3 were relatively smooth and appeared crystalline. The TEM and DFM images clearly show that the fibrous aggregates responsible for physical gelation play an important role in effective quenching. The gelators 1 and 2 form 3D networks of fibrous aggregates in ethanol, resulting in physical gelation due to the trapping of solvent molecules. The xerogel films prepared by drop-casting the ethanol gels have wide surface area compared with those of trans-4-(4-aminostyryl)benzonitrile and compound 3. The widely surfaces 
can successfully come in contact with explosive vapors, resulting in effective quenching.

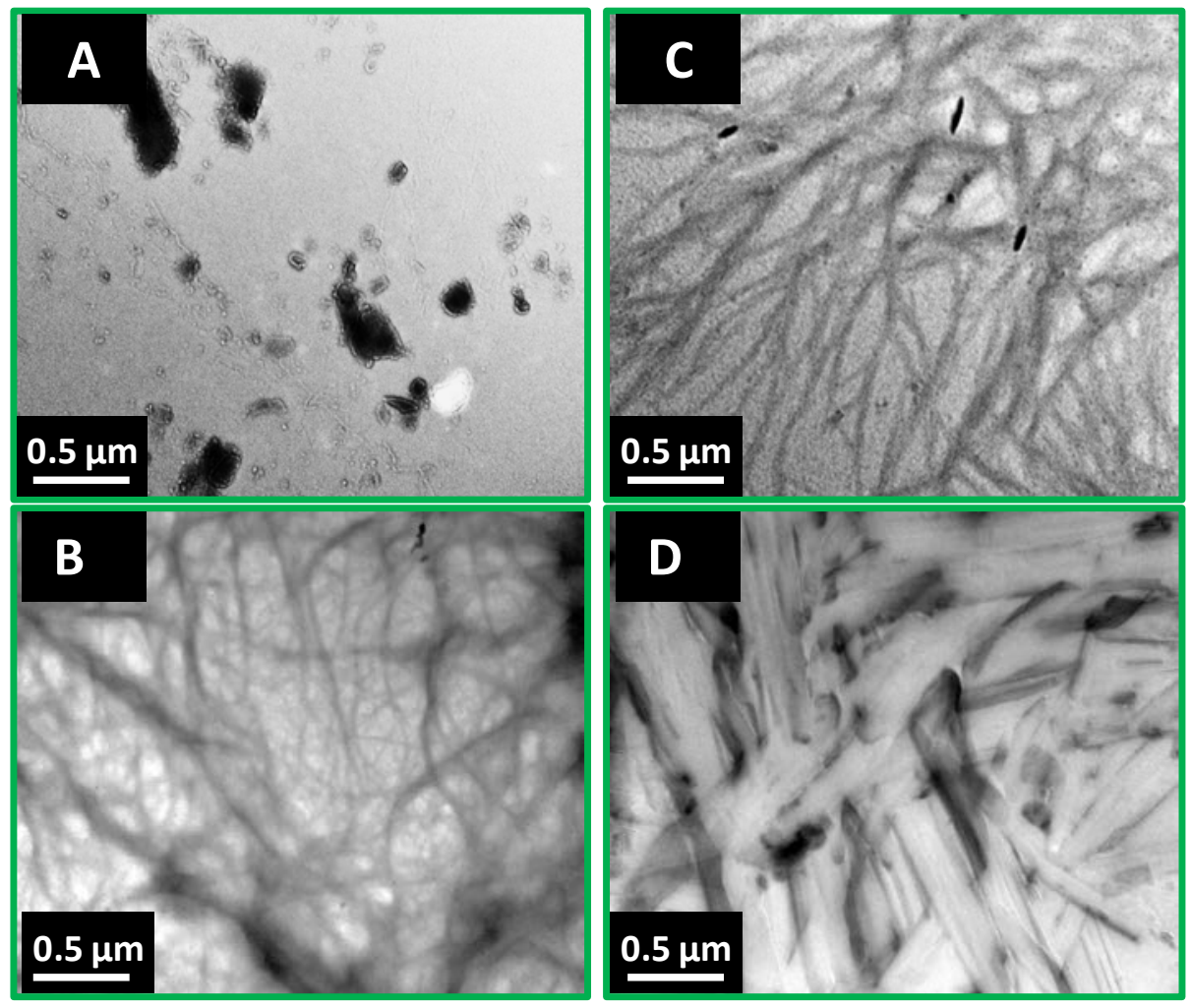

Figure 7. TEM images of trans-4-(4-aminostyryl)benzonitrile (A), 1 (B), 2 (C), and 3 (D) prepared from ethanol solutions $(5.0 \times 10-4 \mathrm{M})$. 


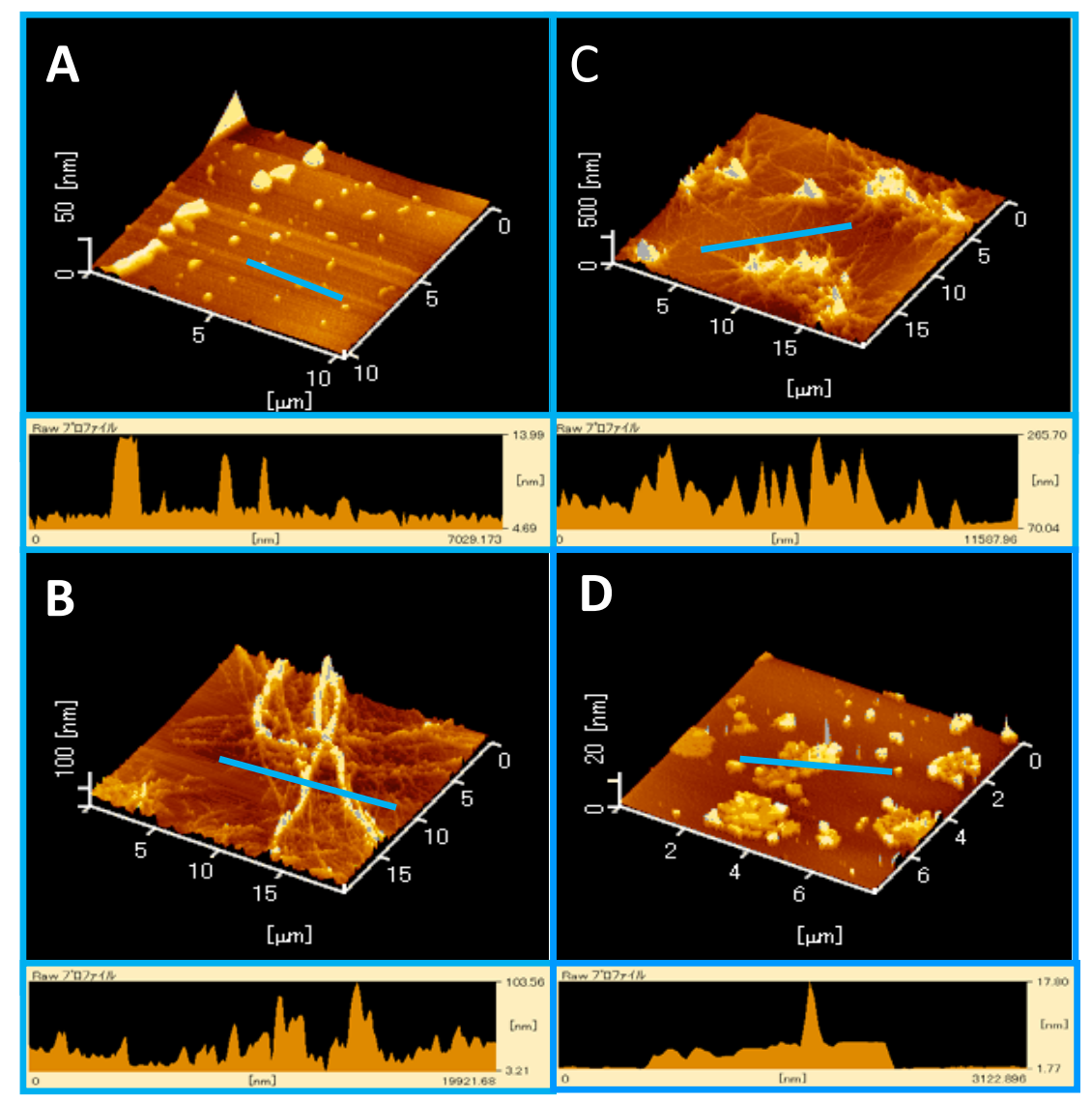

Figure 8. DFM images of trans-4-(4-aminostyryl)benzonitrile (A), 1 (B), 2 (C), and 3 (D) prepared from ethanol solutions $(5.0 \times 10-4 \mathrm{M})$.

The effects of casting solvents on quenching (see Figure 4) can be also interpreted in terms of the differences in film morphology. The xerogel films formed from toluene gel exhibited 3D fibrous networks with widths of several tens of nanometers in TEM and entangled fibrous aggregates with very uneven surfaces in DFM. The film prepared from the chloroform solution of $\mathbf{2}$ was crystalline with a planar surface.

TEM and DFM images of the xerogel film of gelator $\mathbf{4}$, which showed effective quenching upon exposure to RDX, are shown in Figure 9. The formation of dense 3D fibrous networks with average widths of $20 \mathrm{~nm}$, which were responsible for the gelation of solvents, was observed in the TEM images. The fiber diameters were nearly homogeneous. The height profiles of selected regions demonstrate fibers with widths of approximately several hundreds of nanometers and heights ranging 50 to $120 \mathrm{~nm}$, with the fibers rising on the wafer. 

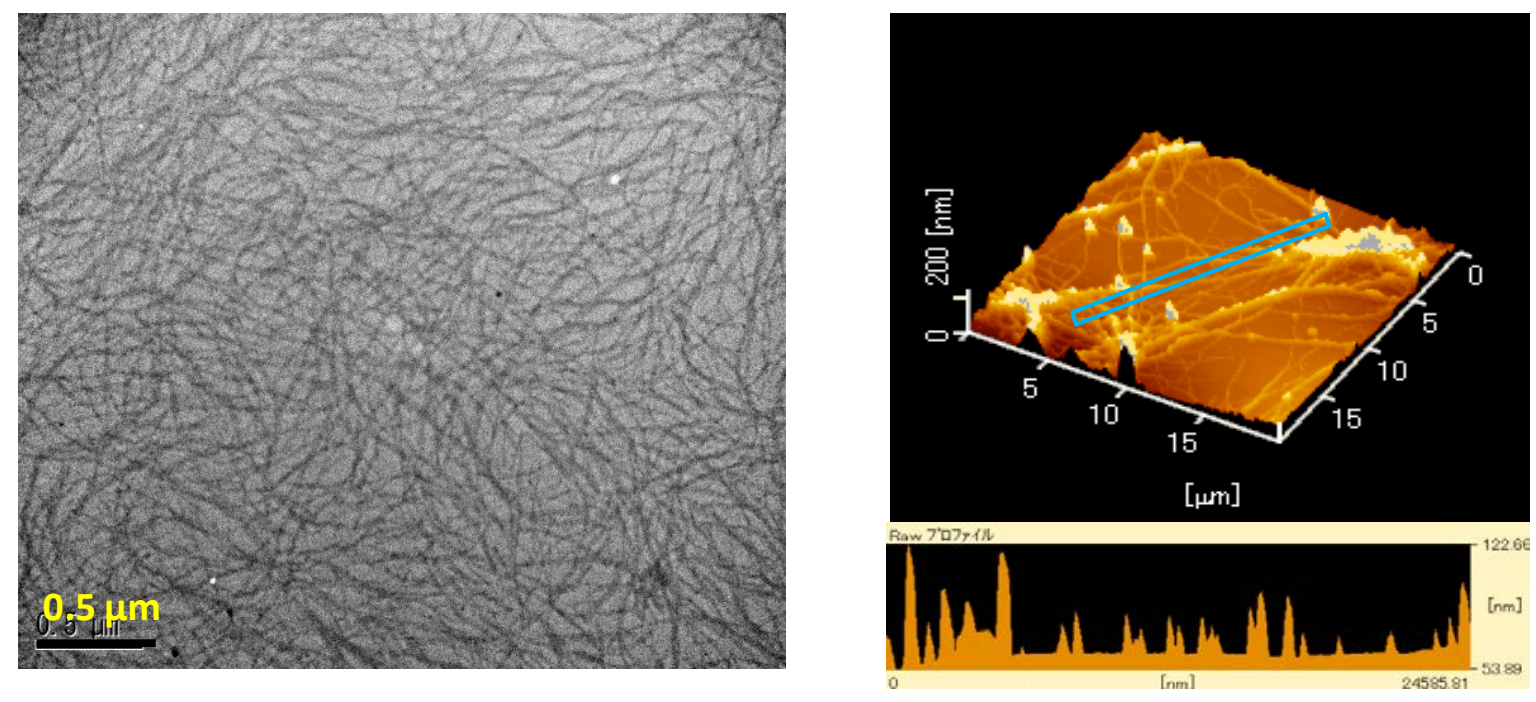

Figure 9. TEM and DFM images of 4 prepared from toluene solution $(5.0 \times 10-4 \mathrm{M})$.

\section{Mechanism for Detecting TNT}

Energy calculations with optimized geometries were carried out using density functional theory at the B3LYP/6-31G* level with respect to trans-4-(4-aminostyryl)benzonitrile and trans-4-(4-aminostyryl)-3,5-bis(trifluoromethyl)benzene. The HOMO and LUMO energies of trans-4-(4-aminostyryl)benzonitrile were -6.67 and $-0.95 \mathrm{eV}$, respectively, whereas those of TNT were calculated to be -8.7 and $-3.8 \mathrm{eV}$, respectively. The photoinduced electron transfer from trans-4-(4-aminostyryl)benzonitrile to TNT is clearly possible, resulting in the fluorescence quenching of the former. The driving force for the photoinduced electron transfer from trans-4-(4-aminostyryl)benzonitrile to TNT of the quencher is $1.66 \mathrm{eV}$. The HOMO and LUMO energies of trans-4-(4-aminostyryl)-3,5bis(trifluoromethyl)benzene were -6.76 and $-0.88 \mathrm{eV}$, respectively. The photoinduced electron transfer from trans-4-(4-aminostyryl)-3,5-bis(trifluoromethyl)benzene to TNT is also possible. HOMO and LUMO energy levels of trans-4-(4-aminostyryl)benzonitrile, trans-4-(4-aminostyryl)-3,5-bis(trifluoromethyl)benzene, and TNT are illustrated in Figure 10. 


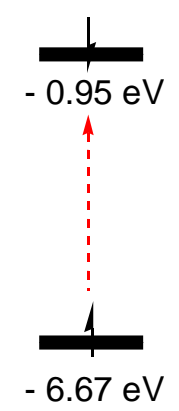

(a)

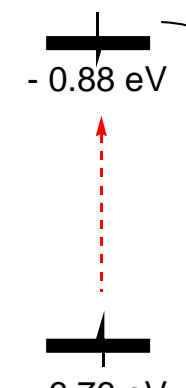

(b)

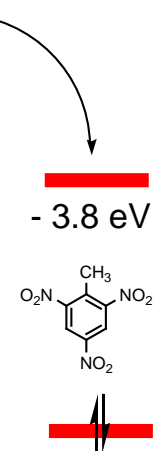

$-8.7 \mathrm{eV}$

Figure 10. HOMO and LUMO energy levels of trans-4-(4aminostyryl)benzonitrile (a), trans-4-(4-aminostyryl)-3,5bis(trifluoromethyl)benzene (b), and TNT

Conclusion: Fluorescent gelators including donor-acceptor-substituted stilbenes were synthesized and their gelation abilities were studied. Fluorescent compounds 1, 2, and 4 could gel ethanol and toluene. Fibrous xerogel films were prepared from the gels on quartz plates, and their abilities to detect explosives were evaluated using fluorescence quenching upon exposure to saturated TNT or RDX vapor. The xerogel film of gelator $\mathbf{1}$ achieved approximately 72\% and 95\% fluorescence quenching after 2 and $20 \mathrm{~min}$ of exposure to saturated TNT, respectively. The fluorescence quenching efficiency decreased distinctly with increasing film thickness. The $72 \%$ quenching efficiency for a xerogel film thickness of $32 \mathrm{~nm}$ was decreased to $21 \%$ for the xerogel film with a thickness of $2590 \mathrm{~nm}$. Despite the very low vapor pressure of saturated RDX, significant decreases in quenching efficiency were observed for xerogel films of gelators 2 and 4; both decreases in quenching efficiency were $\sim 60 \%$ after 20 min of exposure to RDX vapor. The xerogel film morphologies were studied by TEM and DFM images. The xerogel films that exhibited effective fluorescence quenching were found to have 3D fibrous networks with widths of several tens of nanometers and entangled fibrous aggregates with very uneven surfaces. The mechanism of TNT detection was also discussed in terms of the HOMO and LUMO levels.

Acknowledgement: The present research was supported in part by a Grant-in-Aid for Scientific Research (C) (No. 15K05623) from the Ministry of Education, Culture, Sports, Science and Technology of Japan. 
[3] J. H. Esch, B. L. Feringa, Angew. Chem., Int. Ed. 2000, 39, 2263.

[4] L. A. Estroff, A. D. Hamilton, Chem. Rev. 2004, 104, 1201.

[5] P. Dastidar, Chem. Soc. Rev. 2008, 37, 2699.

[6] S. Banerjee, R. K. Das, U. Maitra, J. Mater. Chem. 2009, 19, 6649.

[7] M. Suzuki, K. Hanabusa, Chem. Soc. Rev. 2009, 38, 967.

[8] M. Suzuki, K. Hanabusa, Chem. Soc. Rev. 2010, 39, 455.

[9] J.-L. Li, X.-Y. Liu, Adv. Funct. Mater. 2010, 20, 3196.

[10] G. John, B. V. Shankar, S. R. Jadhav, P. K. Vemula, Langmuir 2010, 26, 17843.

[11] H. Svobodová, V. Noponen, E. Kolehmainen, E. Sievänen, RSC Adv. 2012, 2, 4985.

[12] S. S. Babu, S. Prasanthkumar, A. Ajayaghosh, Angew. Chem., Int. Ed. 2012, 51, 1766.

[13] A. Y.-Y. Tam, V. W.-W. Yam, Chem. Soc. Rev. 2013, 42, 1540.

[14] J. Raeburn, A. Z. Cardoso, D. J. Adams, Chem. Soc. Rev. 2013, 42, 1543.

[15] G. Yu, X. Yan, C. Han, F. Huang, Chem. Soc. Rev. 2013, 42, 6697.

[16] M. D, Segarra-Maset, V. J. Nebot, J. F. Miravet, B. Escuder, Chem. Soc. Rev. 2013, 42, 7086.

[17] S. S. Babu, V. K. Praveen, A. Ajayaghosh, Chem. Rev. 2014, 114, 1973.

[18] D. K. Kumar, J. W. Steed, Chem. Soc. Rev. 2014, 43, 2080.

[19] V. K. Praveen, C. Ranjith, N. Armaroli, Angew. Chem., Int. Ed. 2014, 53, 365.

[20] Y. Lan, M. G. Corradini,aR. G. Weiss, S. R. Raghavanc, M. A. Rogers, Chem. Soc. Rev. 2015, 44, 6035.

[21] X. Li, J. Li, Y. Gao, Y. Kuang, J. Shi, B. Xu, J. Am. Chem. Soc. 2010, 132, 17707.

[22] J. Li, Y.Gao, Y. Kuang, J. Shi, X. Du, H. Wang, Z. Yang, B. Xu, J. Am. Chem. Soc. 2013, 135, 9907.

[23] L. Chronopoulou, A. R. Togna, G. Guarguaglini, G. Masci, F. Giammarco, G. I. Togna, C. Palocci, Soft Matter, 2012, 8, 5784.

[24] B. F. Lin, K. A. Megley, N. Viswanathan, D. V. Krogstad, L. B. Drews, M. J. Kade, Y. Qian, M. V. Tirrell, J. Mater. Chem. 2012, 22, 19447.

[25] K. Murata, M. Aoki, T. Nishi, A. Ikeda, S. Shinkai, Chem. Commun. 1991, 1715.

[26] J. J. D. de Jong, L. N. Lucas, R. M. Kellogg, J. H. van Esch, B. L. Feringa, Science 2004, 304, 278.

[27] J. Chen, W. Wu, and A. J. McNeil, Chem. Commun. 2012, 48, 7310.

[28] P. Xue, Q. Xu, P. Gong, C. Qian, A. Ren, Y. Zhang, and R. Lu, Chem. Commun. 2013, 49, 5838.

[29] Y. Ono, K. Nakashima, M. Sano, Y. Kanekiyo, K. Inoue, J. Hojo, S. Shinkai, Chem. Commun., 1998, 1477.

[30] H. Basit, A. Paul, S. Sen, S. Bhattacharya, Chem.-Eur. J., 2008, 14, 6534.

[31] S. Kobayashi, K. Hanabusa, N. Hamasaki, M. Kimura, H. Shirai, S. Shinkai, Chem. Mater., 2000, 12, 1523.

[32] S. Kobayashi, N. Hamasaki, M. Suzuki, M. Kimura, H. Shirai, K. Hanabusa, J. Am. Chem. Soc., 2002, 124, 6550.

[33] Y. Yang, M. Suzuki, H. Fukui, H. Shirai, K. Hanabusa, Chem. Mater., 2006, 18, 1324.

[34] W. Kubo, S. Kambe, S. Nakade, T. Kitamura, K. Hanabusa, Y. Wada, S. Yanagida, J. Phys. Chem. B 2003, 107, 4374.

[35] S. S. Babu, S. Prasanthkumar, A. Ajayaghosh, Angew. Chem., Int. Ed. 2012, 51, 1766.

[36] K. Hanabusa, K. Hiratsuka, M. Kimura, H. Shirai, Chem. Mater. 1999, 11, 649.

[37] C. Vijayakumar, G. Tobin, W. Schmitt, M.-J. Kim, M. Takeuchi, Chem. Commun. 2010, 46, 874.

[38] Y. Yang, H. Wang, K. Su, Y. Long, Z. Peng, N. Li, F. Liu, J. Mater. Chem. 2011, 21, 11895.

[39] C. Zhang, Y. Che, X. Yang, B. R. Bunes, L. Zang, Chem. Commun. 2010, 46, 5560.

[40] H. Nie, Y. Zhao, M. Zhang, Y. Ma, M. Baumgarten, K. Möllen, Chem. Commun. 2011, 47, 1234.

[41] Z. Ding, Q. Zhao, R. Xing, X. Wang, J. Ding, L. Wang, Y. Han, J. Mater. Chem. C 2013, 1, 786.

[42] K. K. Kartha, S. S. Babu, S. Srinivasan, A. Ajayaghosh, J. Am. Chem. Soc. 2012, 134, 4834.

[43] N. Dey, S. K. Samanta, S. Bhattacharya, ACS Appl. Mater. Interfaces 2013, 5, 8394.

[44] K. Hanabusa, M. Suzuki, Bull. Chem. Soc. Jpn. in press.

[45] V. Papper, D. Pines, G. Likhtenshtein, E. Pines, J. Photochem. Photobiol. A, Chem. 1997, 111, 87. [46] M. Suzuki, R. Yanagida, C. Setoguchi, H. Shirai, K. Hanabusa, J. Polym. Sci., Part A: Polym. Chem. 2008, 46, 353.

[47] M. Suzuki, C. Setoguchi, H. Shirai, K. Hanabusa, Chem.-Eur. J. 2007, 13, 8193.

[48] R. C. Dorey, W. R. Carper, J. Chem. Eng. Data 1984, 29, 93.

[49] G. C. Hale, J. Am. Chem. Soc. 1925, 47, 2754.

[50] W. E. Bachmann, J. Sheehan, J. Am. Chem. Soc. 1949, 71, 1842. 